OPEN ACCESS

Edited by:

Maurizio Acampa,

Siena University Hospital, Italy

Reviewed by:

Svetlana Lorenzano

Sapienza University of Rome, Italy

Anita Ante Arsovska,

Saints Cyril and Methodius University

of Skopje, North Macedonia

Sang-Hwa Lee,

Hallym University, South Korea

*Correspondence:

Jianren Liu

liujr021@sjtu.edu.cn

Jingjing Su

jingjingsu2000@163.com

${ }^{\dagger}$ These authors have contributed equally to this work and share first authorship

Specialty section

This article was submitted to

Stroke,

a section of the journal

Frontiers in Neurology

Received: 04 May 2021

Accepted: 28 July 2021

Published: 19 August 2021

Citation:

Pan H, Lin C, Chen L, Qiao Y Huang P, Liu B, Zhu Y, Su J and Liu J

(2021) Multiple-Factor Analyses of Futile Recanalization in Acute Ischemic

Stroke Patients Treated With

Mechanical Thrombectomy.

Front. Neurol. 12:704088.

doi: 10.3389/fneur.2021.704088

\section{Multiple-Factor Analyses of Futile Recanalization in Acute Ischemic Stroke Patients Treated With Mechanical Thrombectomy}

\author{
Hui Pan ${ }^{1 \dagger}$, Changchun Lin ${ }^{1 \dagger}$, Lina Chen ${ }^{1 \dagger}$, Yuan Qiao ${ }^{1}$, Peisheng Huang ${ }^{1}$, Bin Liu ${ }^{2}$, \\ Yueqi Zhu ${ }^{3}$, Jingjing Su${ }^{1 *}$ and Jianren Liu ${ }^{1 *}$
}

'Department of Neurology, Shanghai Ninth People's Hospital, Shanghai Jiao Tong University School of Medicine, Shanghai, China, ${ }^{2}$ Department of Neurology, Shanghai Minhang Hospital, Fudan University, Shanghai, China, ${ }^{3}$ Department of Interventional Radiology, Shanghai Sixth People's Hospital, Shanghai Jiao Tong University School of Medicine, Shanghai, China

Background and Purpose: Acute ischemic stroke (AIS) is a serious threat to the life and health of middle-aged and elderly people. Mechanical thrombectomy offers the advantages of rapid recanalization, but the response of patients to this treatment varies greatly. This study investigated the risk factors for futile recanalization in AIS patients after thrombectomy through multivariate analyses.

Methods: A retrospective study was conducted in AIS patients with anterior circulation occlusion from a derivation cohort and a validation cohort who underwent thrombectomy and reperfusion defined as a modified Thrombolysis in Cerebral Infarction ( $\mathrm{mTICI}$ ) score of $2 \mathrm{~b} / 3$. Using the modified Rankin Scale (mRS) at 90 days after the operation, the patients were divided into two groups, the meaningful recanalization group ( $m R S \leq 2)$, and the futile recanalization group ( $\mathrm{mRS}>2$ ). Multivariate logistic regression analyses were performed, and the receiver operating characteristic (ROC) curve was used to construct a risk prediction model for futile recanalization. The performance of prediction model was evaluated on the validation cohort.

Results: A total of 140 patients in the derivation cohort were enrolled, 46 patients in the meaningful recanalization group and 94 patients in the futile recanalization group. The two groups were significantly different in age, preoperative National Institute of Health Stroke Scale (NIHSS) score, and collateral circulation ASITN/SIR grade $(P<0.05)$. In multivariate regression analyses, patients' age $\geq 71$, NIHSS $\geq$ 12 , and $A S I T N / S I R \leq 3$ were risk factors for futile recanalization. Hence, an ANA (Age-NIHSS-ASITN/SIR) score scale consisting of age, NIHSS score, and ASITN/SIR grade factors can effectively predict the risk for futile recanalization (area under curve $0.75,95 \% \mathrm{Cl} 0.67-0.83$, specificity $67.4 \%$, and sensitivity $73.4 \%$ ). The proportion of patients with futile recanalization in ANA groups $0,1,2$, and 3 were 21.05, 56.76, 79.03, and 90.91\%, respectively. Furthermore, ANA score scale had also a good performance for predicting futile recanalization on the validation cohort. 
Conclusions: Old age, high baseline NIHSS, and poor collateral circulation are risk factors for futile recanalization in AIS patients treated with thrombectomy. An ANA score that considers age, NIHSS, and collateral ASITN/SIR can effectively predict the risk for futile recanalization. Further studies with a larger sample size are needed to validate the prognostic value of this combined score for futile recanalization.

Keywords: acute ischemic stroke, futile recanalization, mechanical thrombectomy, NIHSS score, collateral circulation

\section{INTRODUCTION}

Acute ischemic stroke (AIS) has become a serious disease burden because of its high mortality and disability (1). The key in treating AIS patients is to reopen the occluded arteries as early as possible. With the technological development of intravascular interventional therapy and the update of clinical guidelines, intravascular mechanical thrombectomy therapy has become widely accepted by clinicians $(2,3)$. However, futile recanalization after mechanical thrombectomy is common in AIS patients $(4,5)$. Futile recanalization is defined as poor clinical outcome, or treatment failure despite adequate vessel recanalization, following endovascular treatment in AIS patients (4). It has been reported that futile recanalization was observed in almost one half of patients after endovascular treatment and the rate of futile recanalization was $40.5-54.5 \%$ (6-9). It may be related to poor collateral circulation, subacute reocclusion, massive hypoperfusion, and/or damage to capillaries and brain autoregulation.

Previous studies have suggested that certain imaging parameters or neural function evaluation methods can be used to predict futile recanalization and identify patients who may not be suitable for endovascular treatment $(6,10)$. Advanced age, severer neurological deficits assessed by the baseline National Institute of Health Stroke Scale (NIHSS), and female gender are considered to be the independent predictors of futile recanalization $(7,11)$. Delayed endovascular treatment and use of general anesthesia have been proposed as the important determinants of unfavorable outcomes in those patients with recanalization $(8,12)$. Baseline Alberta Stroke Program Early Computed Tomography Score (ASPECTS) from the non-contrast CT (NCCT) and CT angiography source images (CTA-SI) are useful in predicting futile recanalization and could be valuable tools for treatment decisions regarding revascularization therapies (13). Similarly, studies employing NCCT to determine the risk factors in AIS patients treated with mechanical thrombectomy have revealed that the presence of leukoaraiosis and brain atrophy is associated with poor outcome in recanalized patients $(14,15)$. Furthermore, another study applying diffusion-weighted magnetic resonance imaging (DWI) to predict futile recanalization has demonstrated that patients with large preintervention DWI lesions in the deep white matter may be poor candidates for endovascular therapy (6). Therefore, the use of functional imaging such as CT and magnetic resonance imaging (MRI) solely, or in combination, provides encouraging results as independent prognostic predictors for futile recanalization $(16,17)$. However, adopting these approaches during clinical practice may not be feasible; it remains unclear due to various issues among studies, including a small number of patients enrolled, and large variation in clinical characteristics and treatment strategies (18).

Studies on risk factors for futile recanalization in the Chinese AIS cohort are scarce. A substantial medical resource is required to identify underlying risks during mechanical thrombectomy treatment. We analyzed the risk factors for futile recanalization in a Chinese AIS cohort after mechanical thrombectomy. We also evaluated and validated whether the combination of these factors would improve prognostic value for the prediction of futile recanalization in such patients.

\section{MATERIALS AND METHODS Study Design and Patients}

This study was a retrospective study and included 2 cohorts of AIS patients from January 2016 to July 2020. A derivation cohort of 140 patients ( 85 males and 55 females) were admitted at the Department of Neurology, Shanghai Ninth People's Hospital, Shanghai Jiao Tong University School of Medicine. A validation cohort of 154 patients (98 males and 56 females) were from the Shanghai Minhang Hospital and Shanghai Sixth People's Hospital. All the patients had undergone mechanical thrombectomy treatment and recanalization in this study. Post-intravascular therapy reperfusion was graded using the modified Thrombolysis in Cerebral Infarction (mTICI) scales. The mTICI scores of $2 \mathrm{~b} / 3$ were considered recanalization after mechanical thrombectomy (19). The mTICI grade was retrospectively confirmed by 2 neuroradiologists blinded to clinical and procedural data. According to the modified Rankin Scale (mRS) score at 90 days after the operation, the patients were divided into the meaningful recanalization group ( $\mathrm{mRS} \leq 2$ points, good prognosis) and the futile recanalization group (mRS $>2$ points, poor prognosis) $(4,20)$.

Further inclusion criteria were as follows: acute anterior large vessel occlusion as assessed via CTA, baseline ASPECTS $\geq 6$ points (21), degree of preoperative neurological deficits assessed by the NIHSS score $\geq 2$ points (22), and mechanical thrombectomy performed within $6 \mathrm{~h}$ of symptom onset without contraindications, or a small infarct core, and moderate-to-good collateral circulation on CT and CTA, or clinical symptoms mismatched with imaging findings (i.e., preoperative NIHSS score showing significant neurological deficits but small infarct 
core area in DWI or CT perfusion) within 6-24h of symptom onset $(17,23,24)$, plus digital subtraction angiography (DSA) showing good collateral circulation. Patients were excluded from the study if they had severe heart, kidney, or liver failure. AIS patients suffering from vasculitis or coagulation disorders, and subjects diagnosed with intracerebral hemorrhage were also excluded. The exclusion criteria for intravenous alteplase thrombolysis were as follows: delayed arrival time $>4.5 \mathrm{~h}$ after stroke onset, previous intracranial hemorrhage, stroke or brain trauma within 3 months, systolic blood pressure of more than $180 \mathrm{mmHg}$, platelet $<100,000 / \mu \mathrm{L}$, glucose level $<2.7 \mathrm{mmol} / \mathrm{L}$, active Partial Thromboplastin Time (aPTT) $>40 \mathrm{~s}$, International Normalized Ratio (INR) > 1.7, and patient/family refusal (25).

This study was approved by the institutional ethics committee (SH9H-2020-T390-2) and informed consent forms were signed based on the study design.

\section{Clinical Assessments}

Clinical data regarding sex, age, history of stroke or transient ischemic attack (TIA), and vascular disease risk factors (e.g., smoking, drinking, hypertension, diabetes, coronary heart disease, and atrial fibrillation) were recorded. Stroke subtype was based on the Trial of ORG 10172 in Acute Stroke Treatment (TOAST) classification, such as large-artery atherosclerosis (LAA), cardioembolism (CE), stroke of other determined cause (ODC), and stroke of undetermined etiology (SUE). Neurological deficits were evaluated using the preoperative NIHSS as the baseline for disability assessment. Imaging parameters such as the baseline ASPECTS based on CT scans, the American Society of Intervention and Therapeutic Neuroradiology/Society of Interventional Radiology (ASITN/SIR) grade of collateral circulation based on DSA, the arterial occlusive lesion (AOL) grade for recanalization after intravascular therapy and the modified Fazekas scale for leukoaraiosis were also recorded. All imaging data were assessed by 2 experienced neuroradiologists blinded to the clinical information.

\section{Endovascular Treatment}

The patients were immediately transported to the neuroradiography suite. They received local or general anesthesia with endotracheal intubation at the discretion of the anesthesiologists. Endovascular treatment was performed by 2 experienced neurointerventionalists. Briefly, the patient's femoral artery was punctured and DSA was performed to determine the occlusion site. Using coaxial catheter technology, the tip of micro-catheter (Rebar 21/27, EV3, USA) was put at the distal end of the occluded artery under the guidance of a micro-guide wire (0.014 in Synchro-14, Stryker, U.S.), then the micro-guide wire was withdrawn. Subsequently, the whole cerebral angiography confirmed that the micro-catheter was in the arterial lumen. The Solitaire ${ }^{\mathrm{TM}} \mathrm{AB}$ embolization stents (EV3, USA) with diameters of 4-6 $\mathrm{mm}$ and lengths of $15-30 \mathrm{~mm}$ were selected according to the diameter of the occluded blood vessels. The stent was introduced to the distal end of the occlusion through the micro-catheter and then was released. Then the contrast agents for visualization were injected, and negative pressure was applied to the catheter to slowly withdraw the stent and remove the thrombus. Recanalization of the main arteries and branches was confirmed by re-examination, indicating that the thrombus was successfully removed. The number of stent retriever passes per procedure was generally no more than 4 times. In the event that residual stenosis $>50 \%$ remained after the procedure, either balloon dilation and/or stent implantation angioplasty were performed after comprehensive consideration. At the end of the procedure, the patients were transported to the neurological intensive care unit for standard stroke care in the charge of neurologists.

\section{Statistical Analyses}

$\mathrm{R}$ software (version number 3.6.2; packages table 1, tidyverse, pROC and rms) and SPSS software (version number 22.0; IBM company) were used for statistical analyses. Quantitative data were described as medians and interquartile ranges (IQR) and categorical variables were described as numbers and percentages of cases. Analyses of the quantitative differences between two groups were compared using the Mann-Whitney U test. Chisquare or Fisher's exact tests were used in statistical analyses of categorical variables. The test-retest reliability was assessed by calculating Intraclass Correlation Coefficients (ICC) for the imaging parameters. An ICC between 0.6 and 0.8 , and higher was regarded as good and excellent, respectively (26). Using futile recanalization as the dependent variable, general clinical data (sex, age), neurological deficits assessment (NIHSS), and functional imaging data (ASPECTS, ASITN/SIR and AOL grades) were used as independent variables. Briefly, univariate logistic regression analyses were used to identify risk factors for futile recanalization, and the odds ratio (OR) and 95\% confidence interval (CI) were described. Subsequently, multivariate logistic regression analyses were used to identify independent risk factors for futile recanalization after adjustment of potential confounders $(P<0.05)$. To evaluate prediction values of variables solely, or in combination, receiver operating characteristic (ROC) curves were plotted. The OR and 95\% CI were calculated. $P$-value $<0.05$ was considered statistically significant.

\section{RESULTS}

\section{Comparison of Clinical Characteristics in the Study Population}

In all, 140 AIS patients in the derivation cohort with anterior circulation occlusion who were recanalized after mechanical thrombectomy and evaluated by postoperative mTICI with scores of $2 b / 3$ were included in the analyses (19). The clinical data of all the patients were presented in Table 1. For the continuous variables reported as median values, the numbers in round brackets were IQR. The postoperative 90-day mRS was used to assess neurological function as an indicator of the effects of endovascular treatment $(4,20)$. The subjects were divided into two groups: meaningful recanalization group ( $\mathrm{mRS} \leq 2$ points, good prognosis) and futile recanalization group ( $\mathrm{mRS}>2$, poor prognosis) (Table 1). Overall, 94 patients (67.1\%) were in the futile recanalization group, of which 54 were male $(57.4 \%, P>$ 0.05 compared to the meaningful recanalization group), with a median age of $71.0(62,80)$ years $(P=0.004$ compared to the 
TABLE 1 | Comparison of clinical characteristics between meaningful recanalization and futile recanalization groups after mechanical thrombectomy in AIS patients.

\begin{tabular}{|c|c|c|c|c|}
\hline Characteristics & $\begin{array}{c}\text { Total } \\
(n=140)\end{array}$ & $\begin{array}{l}\text { Meaningful recanalization } \\
\qquad(\mathrm{mRS} \leq 2, n=46)\end{array}$ & $\begin{array}{l}\text { Futile recanalization } \\
\qquad(\mathrm{mRS}>2, n=94)\end{array}$ & $P$-values \\
\hline Sex (male), $n$ (\%) & $85(60.7 \%)$ & $31(67.4 \%)$ & $54(57.4 \%)$ & 0.343 \\
\hline Age, year, median (IQR) & $67.5(59-78)$ & $64.0(55-72)$ & $71.0(62-80)$ & 0.004 \\
\hline Smoking, $n(\%)$ & $42(30.0 \%)$ & 17 (37.0\%) & $25(26.6 \%)$ & 0.289 \\
\hline Drinking, $n(\%)$ & $26(18.6 \%)$ & $11(23.9 \%)$ & 15 (16.0\%) & 0.365 \\
\hline Hypertension, n (\%) & 83 (59.3\%) & $24(52.2 \%)$ & $59(62.8 \%)$ & 0.310 \\
\hline Diabetes, $n(\%)$ & $37(26.4 \%)$ & $11(23.9 \%)$ & $26(27.7 \%)$ & 0.789 \\
\hline History of stroke or TIA, $n(\%)$ & $21(15.0 \%)$ & $7(15.2 \%)$ & 14 (14.9\%) & 1.000 \\
\hline Coronary heart disease, $n(\%)$ & $21(15.0 \%)$ & $7(15.2 \%)$ & 14 (14.9\%) & 1.000 \\
\hline Atrial fibrillation, $n(\%)$ & $58(41.4 \%)$ & $12(26.1 \%)$ & 46 (48.9\%) & 0.017 \\
\hline TOAST, $n(\%)$ & & & & 0.323 \\
\hline LAA & $84(60.0 \%)$ & 32 (69.6\%) & $52(55.3 \%)$ & \\
\hline CE & $40(28.6 \%)$ & $9(19.6 \%)$ & $31(33.0 \%)$ & \\
\hline ODC & $1(0.7 \%)$ & $0(0.0 \%)$ & $1(1.1 \%)$ & \\
\hline SUE & $15(10.7 \%)$ & 5 (10.9\%) & $10(10.6 \%)$ & \\
\hline Baseline SBP, mmHg, median (IQR) & $150(132-166)$ & $145(127-160)$ & $150(136-172)$ & 0.154 \\
\hline Baseline DBP, mmHg, median (IQR) & $80(73-94)$ & $81(78-94)$ & $80(71-93)$ & 0.544 \\
\hline Glucose, mmol/L, median (IQR) & $7.9(6.4-9.2)$ & $7.6(6.9-8.9)$ & $8.0(6.3-9.3)$ & 0.811 \\
\hline LDL-C, mmol/L, median (IQR) & $2.89(2.24-3.51)$ & $3.23(2.35-3.62)$ & $2.62(2.21-3.42)$ & 0.176 \\
\hline Baseline NIHSS, median (IQR) & $14(10-19)$ & $11(8-15)$ & $16(12-20)$ & $<0.001$ \\
\hline Baseline ASPECTS, median (IQR) & $9(8-10)$ & $9(8-9)$ & $8(8-10)$ & 0.381 \\
\hline DSA ASITN/SIR grade, median (IQR) & $3(2-3)$ & $3(2-4)$ & $2(2-3)$ & $<0.001$ \\
\hline \multicolumn{5}{|l|}{ Baseline AOL grade, $n(\%)$} \\
\hline 0 & $97(69.3 \%)$ & 28 (60.9\%) & $69(73.4 \%)$ & 0.244 \\
\hline 1 & $22(15.7 \%)$ & $11(23.9 \%)$ & $11(11.7 \%)$ & \\
\hline 2 & $20(14.3 \%)$ & $7(15.2 \%)$ & $13(13.8 \%)$ & \\
\hline 3 & $1(0.7 \%)$ & $0(0.0 \%)$ & $1(1.1 \%)$ & \\
\hline Fazekas scale, median (IQR) & $0(0-0.5)$ & $0(0-1)$ & $0(0-0.5)$ & 0.735 \\
\hline \multicolumn{5}{|l|}{ Occlusion site, $n$ (\%) } \\
\hline Middle cerebral artery & $109(80.1 \%)$ & $35(81.4 \%)$ & $74(79.6 \%)$ & 0.804 \\
\hline Internal carotid artery & $44(32.4 \%)$ & $19(44.2 \%)$ & 25 (26.9\%) & 0.045 \\
\hline Intravenous thrombolysis, $n(\%)$ & $51(36.4 \%)$ & $15(32.6 \%)$ & $36(38.3 \%)$ & 0.638 \\
\hline Hemorrhage transformation, $n(\%)$ & $50(35.7 \%)$ & $12(26.1 \%)$ & $38(40.4 \%)$ & 0.129 \\
\hline Number of passes per procedure, median (IQR) & $1(1-6)$ & $1(1-5)$ & $1(1-6)$ & 0.362 \\
\hline Later than $6 \mathrm{~h}$ from onset to puncture, $n(\%)$ & $52(38.0 \%)$ & $20(47.6 \%)$ & $32(33.7 \%)$ & 0.121 \\
\hline Time from onset to puncture, hour, median (IQR) & $5.5(4.0-7.5)$ & $5.7(4.2-8.3)$ & $5.3(3.9-7.4)$ & 0.253 \\
\hline Time from arrival to puncture, hour, median (IQR) & $2.4(1.7-3.3)$ & $2.7(2.1-3.8)$ & $2.3(1.5-3.2)$ & 0.038 \\
\hline Time from onset to recanalization, hour, median (IQR) & $7.4(5.7-9.1)$ & $7.6(6.0-9.7)$ & $7.0(5.7-9.0)$ & 0.391 \\
\hline Procedure time, hour, median (IQR) & $1.7(1.3-2.3)$ & $1.5(1.2-2.3)$ & $1.8(1.3-2.3)$ & 0.288 \\
\hline
\end{tabular}

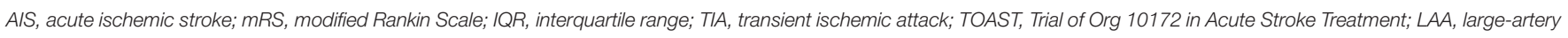

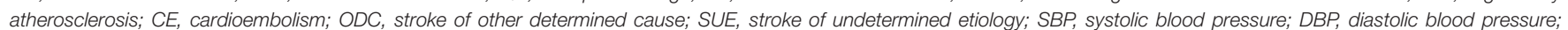

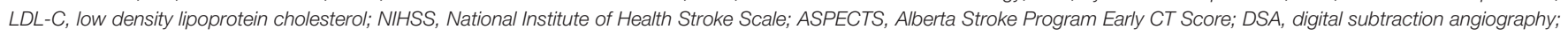
ASITN/SIR, American Society of Intervention and Therapeutic Neuroradiology/Society of Interventional Radiology; AOL, arterial occlusive lesion.

meaningful recanalization group), 46 patients $(48.9 \%, P=0.017)$ with atrial fibrillation, and baseline NIHSS score of $16(12,20)(P$ $<0.001)$. There was also a statistical difference in the ASITN/SIR grade of DSA collateral circulation between the two groups $(P<$ 0.001). As shown in Table 1 , there were no significant differences in other clinical and imaging features, including smoking, drinking, hypertension, diabetes, coronary heart disease, stroke or TIA history, TOAST classification, baseline blood pressure, ASPECTS, and AOL classification $(P>0.05)$. The ICC values for the imaging parameters were reported in Table 2, which were acceptable and ranged from ICC $=0.63-0.96$.

\section{Risk Factors for Futile Recanalization in AIS Patients Treated With Mechanical Thrombectomy}

To identify the risk factors for futile recanalization in AIS patients after mechanical thrombectomy, we conducted univariate logistic 
TABLE 2 | Test-retest intraclass correlation coefficients and 95\% confidence intervals for the imaging parameters.

\begin{tabular}{|c|c|c|}
\hline & ICC (95\% CI) & $P$-values \\
\hline ASPECTS & $0.81(0.69-0.88)$ & 0.000 \\
\hline ASITN/SIR grade & $0.73(0.65-0.80)$ & 0.000 \\
\hline AOL grade & $0.88(0.84-0.92)$ & 0.000 \\
\hline $\mathrm{mTICl}$ grade & $0.63(0.52-0.72)$ & 0.000 \\
\hline Fazekas scale & $0.96(0.95-0.97)$ & 0.000 \\
\hline
\end{tabular}

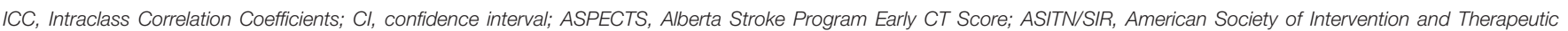
Neuroradiology/Society of Interventional Radiology; AOL, arterial occlusive lesion; mTICl, modified Thrombolysis in Cerebral Infarction.

TABLE 3 | Univariate and multivariate logistic regression analyses of risk factors for futile recanalization after mechanical thrombectomy in AIS patients.

\begin{tabular}{|c|c|c|c|c|}
\hline & \multicolumn{2}{|c|}{ Univariate } & \multicolumn{2}{|c|}{ Multivariate* } \\
\hline & Crude OR (95\% Cl) & $P$-values & Adjusted OR (95\% Cl) & $P$-values \\
\hline Sex & $1.53(0.73-3.21)$ & 0.259 & & \\
\hline Age & $2.36(1.35-4.13)$ & 0.003 & $2.14(1.09-4.20)$ & 0.028 \\
\hline Smoking & $0.62(0.29-1.31)$ & 0.211 & & \\
\hline Drinking & $0.60(0.25-1.45)$ & 0.258 & & \\
\hline Hypertension & 0.65 (0.32-1.32) & 0.232 & & \\
\hline Diabetes & $1.22(0.54-2.75)$ & 0.637 & & \\
\hline History of stroke or TIA & $0.97(0.36-2.61)$ & 0.960 & & \\
\hline Coronary heart disease & $0.97(0.36-2.61)$ & 0.960 & & \\
\hline Atrial fibrillation & $2.72(1.25-5.88)$ & 0.011 & $2.54(0.93-6.92)$ & 0.069 \\
\hline Baseline SBP & $1.43(0.89-2.28)$ & 0.141 & & \\
\hline Baseline DBP & $0.93(0.58-1.48)$ & 0.754 & & \\
\hline Glucose & $1.07(0.74-1.56)$ & 0.714 & & \\
\hline LDL-C & $0.74(0.46-1.20)$ & 0.229 & & \\
\hline Baseline NIHSS & $2.22(1.31-3.75)$ & 0.003 & 2.25 (1.29-3.94) & 0.005 \\
\hline Baseline ASPECTS & $0.69(0.38-1.25)$ & 0.221 & & \\
\hline DSA ASITN/SIR grade & $0.35(0.21-0.57)$ & $<0.001$ & $0.34(0.20-0.58)$ & $<0.001$ \\
\hline Baseline AOL grade & $0.82(0.52-1.29)$ & 0.390 & & \\
\hline Intravenous thrombolysis & $1.28(0.61-2.70)$ & 0.512 & & \\
\hline Hemorrhage transformation & $1.99(0.92-4.34)$ & 0.807 & & \\
\hline Time from onset to puncture & $0.88(0.62-1.26)$ & 0.496 & & \\
\hline Time from onset to recanalization & $0.94(0.66-1.32)$ & 0.711 & & \\
\hline Procedure time & $1.31(0.80-2.14)$ & 0.287 & & \\
\hline
\end{tabular}

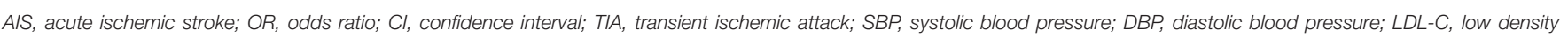

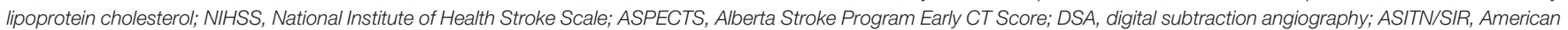

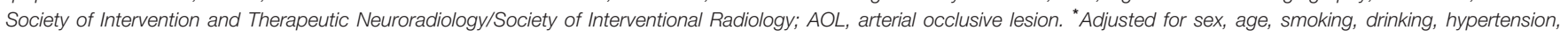
diabetes, history of stroke or TIA, coronary heart disease, atrial fibrillation, and TOAST.

regression analyses. As shown in Table 3, patient age (OR: 2.36, 95\% CI: $1.35-4.13, P=0.003$ ), atrial fibrillation (OR: 2.72 , 95\% CI: $1.25-5.88, P=0.011)$ and high baseline NIHSS score (OR: 2.22, 95\% CI: 1.31-3.75, $P=0.003$ ) were independent risk factors, while high ASITN/SIR grade (OR: 0.35, 95\% CI: $0.21-$ $0.57, P<0.001)$ was a protective factor for futile recanalization. After further adjustment of confounders (including sex, age, smoking, drinking, hypertension, diabetes, history of stroke or TIA, coronary heart disease, atrial fibrillation, and TOAST) in multivariate logistic regression analyses, we found that advanced age (OR: $2.14,95 \% \mathrm{CI}: 1.09-4.20, P=0.028$ ) and high baseline NIHSS score (OR: 2.25, 95\% CI: 1.29-3.94, $P=0.005$ ) were still independent risk factors, while high ASITN/SIR grade (OR: 0.34, 95\% CI: $0.20-0.58, P<0.001)$ was still an independent protective factor for futile recanalization (Table 3 ).

\section{Predictors of Futile Recanalization in AIS Patients Treated With Mechanical Thrombectomy}

Using the ROC curves from the above logistic regression analyses in futile recanalization, we evaluated the predictive efficacy of age, preoperative NIHSS score, and DSA ASITN/SIR grade. We found 


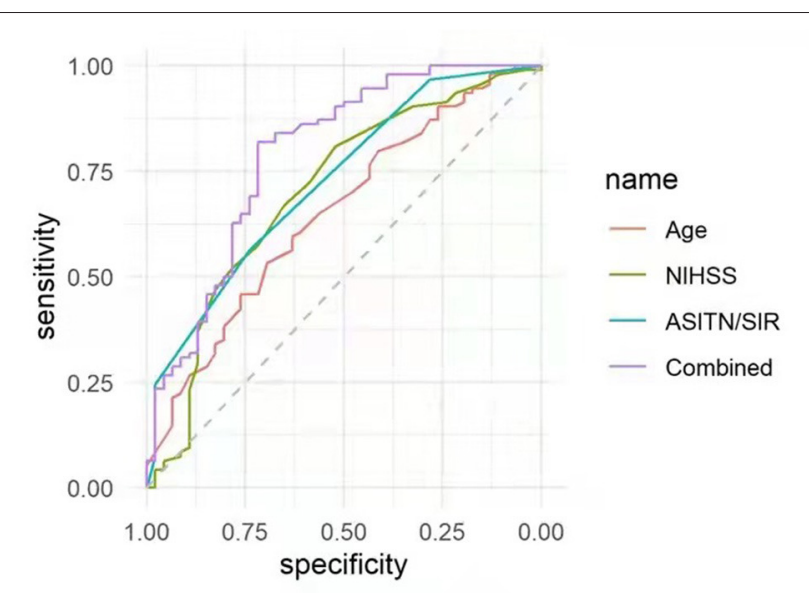

FIGURE 1 | ROC curves were applied to analyze the efficacy of age, preoperative NIHSS score, and ASITN/SIR grade solely, or in combination, for prediction of futile recanalization in AIS patients treated with mechanical thrombectomy. NIHSS, National Institute of Health Stroke Scale; ASITN/SIR, American Society of Intervention and Therapeutic Neuroradiology/Society of Interventional Radiology; ROC, receiver operating characteristic; AIS, acute ischemic stroke.

TABLE 4 | ANA score system derived from a combination of age, preoperative NIHSS score and ASITN/SIR grade in AIS patients with mechanical thrombectomy.

\begin{tabular}{llc}
\hline & Cutoff-point & Score \\
\hline Age, year & $<71$ & 0 \\
& $\geq 71$ & 1 \\
Baseline NIHSS & $<12$ & 0 \\
DSA ASITN/SIR grade & $\geq 12$ & 1 \\
& $>3$ & 0 \\
ANA score & $\leq 3$ & 1 \\
\hline
\end{tabular}

ANA, Age-NIHSS-ASITN/SIR; NIHSS, National Institute of Health Stroke Scale; ASITN/SIR, American Society of Intervention and Therapeutic Neuroradiology/Society of Interventional Radiology; AIS, acute ischemic stroke; DSA, digital subtraction angiography.

that the area under curve (AUC) for age was 0.65 (95\% CI: 0.550.75 ), and the cut-off value was $\geq 71$ years old (specificity $69.6 \%$, and sensitivity 53.2\%). The AUC for baseline NIHSS was 0.70 (95\% CI: $0.60-0.80$ ), and the cut-off value was $\geq 12$ (specificity $52.2 \%$, and sensitivity $80.9 \%$ ). The AUC for ASITN/SIR grade was 0.73 (95\% CI: $0.64-0.81$ ), and the cut-off value was $\leq 3$ (specificity $73.9 \%$, and sensitivity $56.4 \%$ ). Furthermore, the combination of age, NIHSS score, and ASITN/SIR grade had the highest AUC (0.75; 95\% CI: $0.65-0.84)$, the specificity was $67.4 \%$, and the sensitivity was $79.8 \%$ (Figure 1).

\section{ANA Score by Combined Parameter Analyses for Evaluating the Risk for Futile Recanalization in AIS Patients}

Based on the above results, we integrated age, baseline NIHSS score, and collateral circulation ASITN/SIR grade, and constructed a prognosis after mechanical thrombectomy for
AIS patients. Then the scoring index, ANA (Age-NIHSSASITN/SIR), was used to evaluate the risk for futile recanalization in those patients (Table 4).

We divided the 140 patients into four groups according to the ANA score 0-3, and compared the distribution of 90-day mRS scores in these groups. Patients in the ANA score 0 group had the lowest rate of futile recanalization ( $\mathrm{mRS}>2$ ), which was $21.05 \%$, while patients in the ANA score 3 group had 90 -day mRS > 2, which accounted for the highest rate (90.91\%) and risk (OR: 50.07, 95\% CI: 7.74-495.93) of futile recanalization (Figure 2; Table 5).

Furthermore, we used the ROC curve analyses of ANA score to evaluate the predictive efficacy of futile recanalization in AIS patients. The AUC was 0.75 (95\% CI: 0.67-0.83), the specificity was $67.4 \%$, and the sensitivity was $73.4 \%$ (Figure 3A). Among the four ANA score groups, ANA $=0$ group predicted the lowest risk for futile recanalization, and ANA $=3$ group predicted the highest risk for futile recanalization (Figure 3B).

We also performed the ROC curve analyses of ANA score to evaluate the performance of prediction model for futile recanalization on the validation cohort. The AUC was 0.783 (95\% CI: $0.711-0.855)$, the specificity was $61.6 \%$, and the sensitivity was $81.5 \%$ (Figure 4).

\section{DISCUSSION}

We explored the risk factors for futile recanalization in AIS patients treated with mechanical thrombectomy. In addition, we constructed a novel scoring system for predicting this risk by integrating age, baseline NIHSS score, and DSA collateral circulation ASITN/SIR grade. We were also able to effectively predict the risk for futile recanalization by using the scoring system on the validation cohort. The combination of these three independent factors increased the complementary value for prediction of futile recanalization in these patients.

Despite progress in intravascular interventional techniques in recent years, futile recanalization continues to be a serious clinical problem during the implementation of mechanical thrombectomy. In our cohort, we demonstrated that the rate of meaningful recanalization was only $32.9 \%$, while the rate of futile recanalization was $67.1 \%$ in AIS patients after mechanical thrombus removal and recanalization. By contrast, some international randomized controlled trials (RCTs) for endovascular treatment of AIS patients have reported that the proportion of meaningful recanalization is about $47.5 \%$ with strict screening of patients before operation (27-29). Therefore, the development of patients' screening criteria for mechanical thrombectomy is expected to be of great clinical significance for the improvement of meaningful recanalization.

A reliable predictor for futile recanalization is necessary to identify patients who would not benefit from mechanical recanalization. Our study demonstrated that the risk for futile recanalization was higher in our AIS cohort with NIHSS scores $\geq 12$. It is generally believed that the most effective tool for predicting patients with large vessel occlusion 


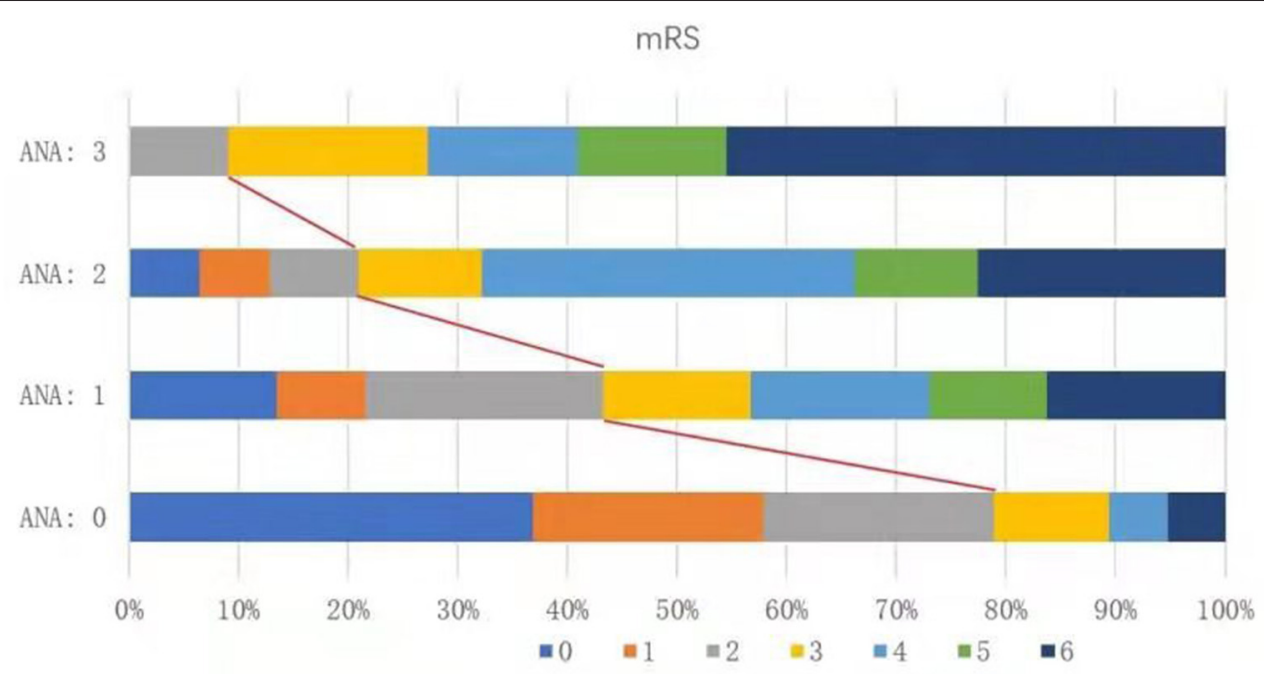

FIGURE 2 | Proportion of different functional outcomes by mRS at 90 days post-operation among groups with different ANA scores in AIS patients treated with mechanical thrombectomy. mRS, modified Rankin Scale; ANA, Age-NIHSS-ASITN/SIR; AIS, acute ischemic stroke.

TABLE 5 | Analyses of ANA score for the risk for futile recanalization in AIS patients treated with mechanical thrombectomy.

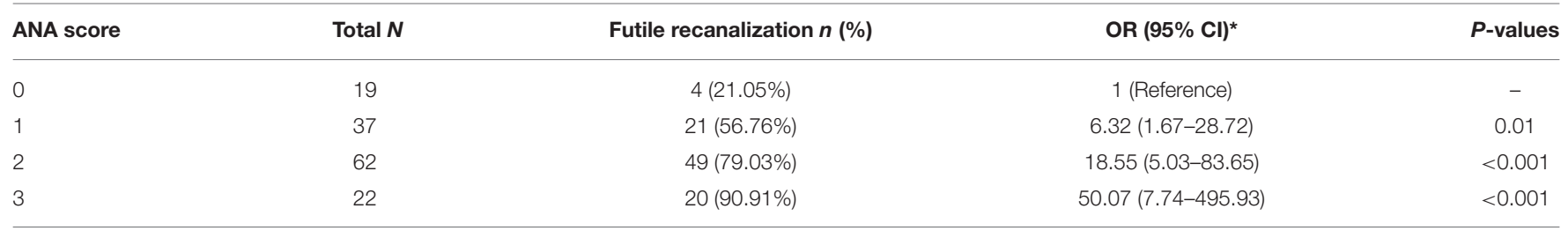

ANA, Age-NIHSS-ASITN/SIR; AIS, acute ischemic stroke; OR, odds ratio; Cl, confidence interval. "Adjusted for sex, age, smoking, drinking, hypertension, diabetes, history of stroke or TIA, coronary heart disease, atrial fibrillation, and TOAST.
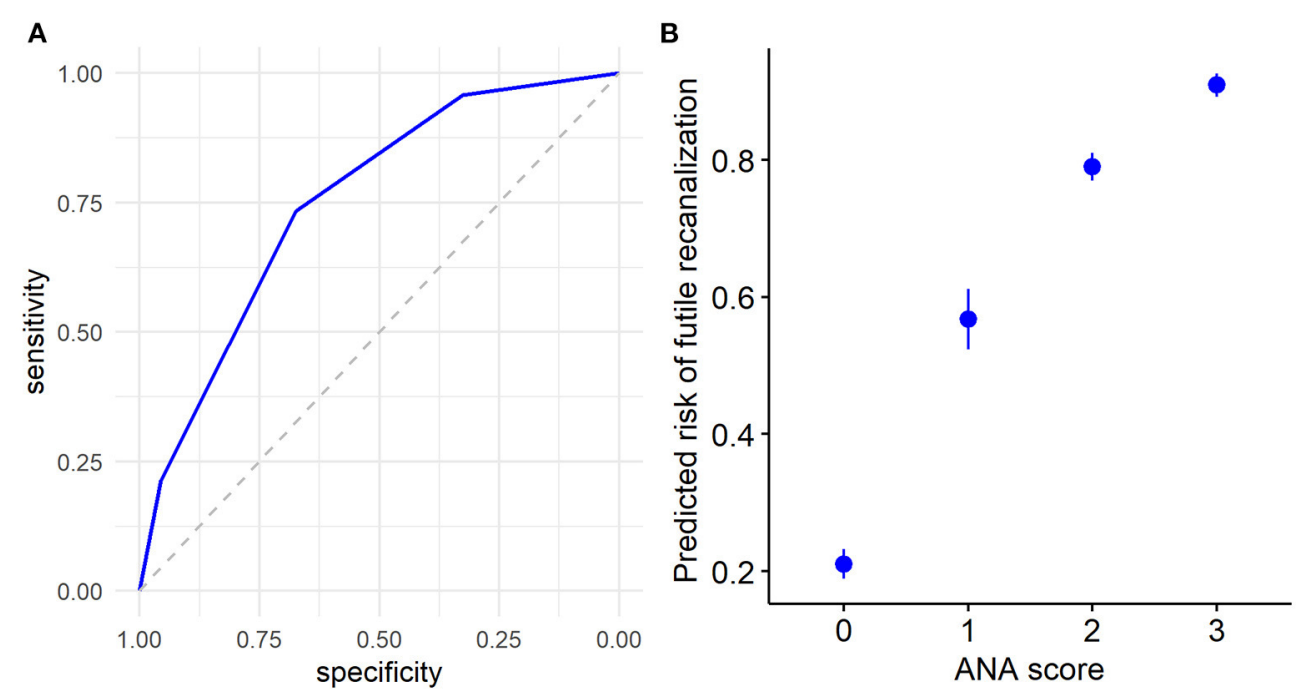

FIGURE 3 | The predicted efficacy of ANA score for futile recanalization in AIS patients treated with mechanical thrombectomy. (A) The ROC curve was applied to analyze the efficacy of ANA score in prediction of futile recanalization. (B) Predicted probability $95 \% \mathrm{Cl}$ of ANA score for futile recanalization in AIS patients. ANA, Age-NIHSS-ASITN/SIR; AIS, acute ischemic stroke; ROC, receiver operating characteristic; Cl, confidence interval. 
A

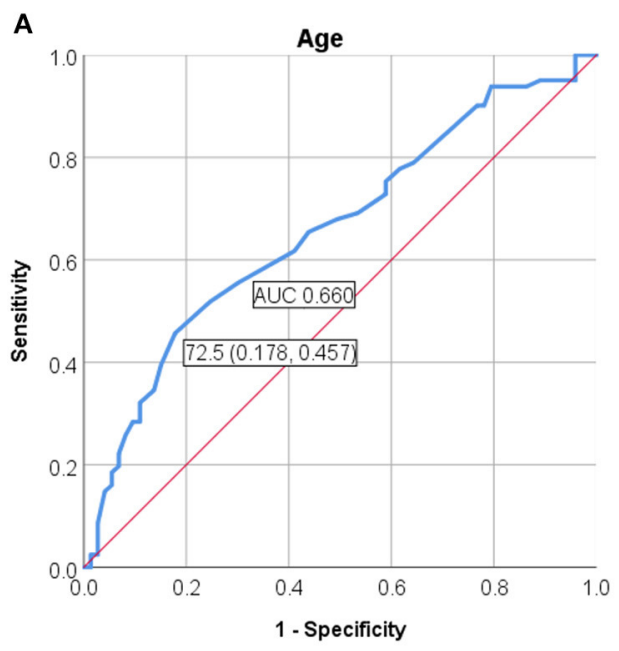

C

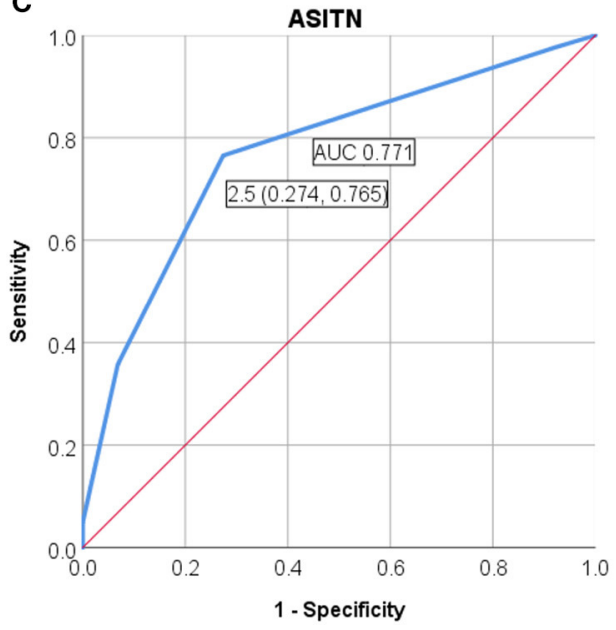

B

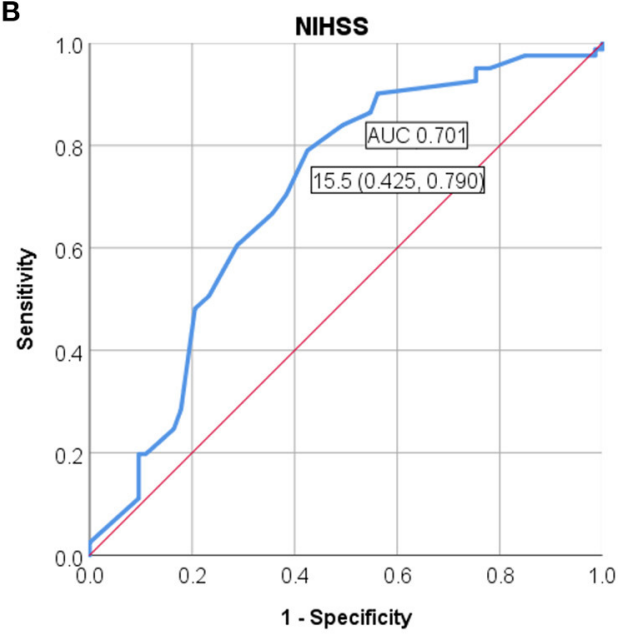

D

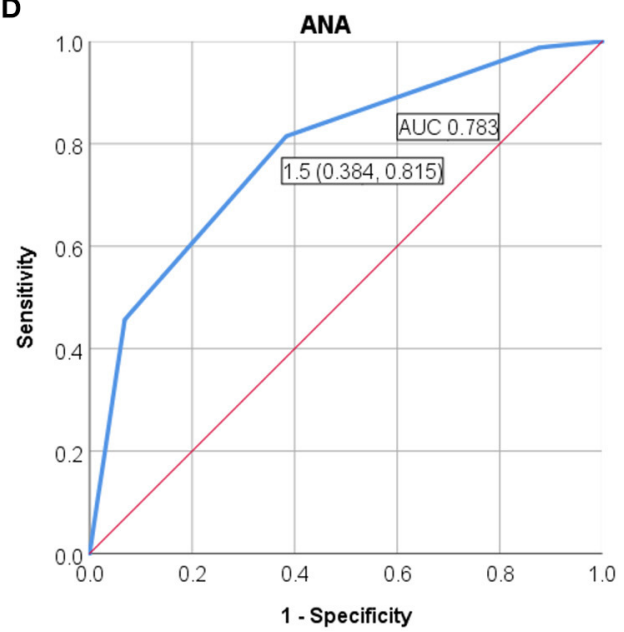

FIGURE 4 | ROC curves were applied to analyze the efficacy of (A) age, (B) preoperative NIHSS score, and (C) ASITN/SIR grade solely, or (D) ANA score, for prediction of futile recanalization in AIS patients with thrombectomy on the validation cohort. NIHSS, National Institute of Health Stroke Scale; ASITN/SIR, American Society of Intervention and Therapeutic Neuroradiology/Society of Interventional Radiology; ANA, Age-NIHSS-ASITN/SIR; AUC, area under curve; ROC, receiver operating characteristic; AIS, acute ischemic stroke.

is the NIHSS score after the initial diagnosis of AIS by neurologists and emergency physicians $(30,31)$. In a metaanalysis, it was found that the best balance of sensitivity and specificity was achieved when the NIHSS score was $\geq 10$ to predict vascular occlusion (32). High baseline NIHSS score (NIHSS score $>10$ ), older age (age $>70$ years old), and delay in treatment were predictors of poor prognosis after complete recanalization $(13,14)$. Similarly, data from our patients older than 71 years old were at increased risk for futile recanalization.

In other studies, imaging parameters based on CT, DSA, and MRI have been tested and used either alone or in combination to select patients suitable for mechanical thrombectomy, and to improve the probability of meaningful recanalization $(6,33)$. A series of RCT studies have reported satisfactory positive results using strict imaging indicators to screen suitable patients $(22,34)$. A number of studies have also shown that collateral flow assessed by DSA can predict response to endovascular therapy for AIS and the status of angiographic collateral blood flow can assist in the decision-making of mechanical thrombectomy treatment (35). DSA has become the gold standard for evaluating cerebral collateral blood flow (36). However, there is no research on collateral flow as a predictor for futile recanalization after endovascular treatment until now. Here, we aimed to determine the predictive value of real status of cerebral collateral blood flow in futile recanalization. In our study, we found that patients with a good collateral circulation state (ASITN/SIR grade $>3$ ) before endovascular treatment had a low risk for futile recanalization. Studies have reported that good collateral circulation state is 
significantly related to the final smaller infarct core volume, lower hemorrhage transformation rate, and lower mortality (37). However, evaluation of ASITN/SIR grade depends on the proficiency and experience of the technical operator. In recent years, with the development of non-invasive angiography technology, some studies have adopted methods including multiphase CT perfusion (MCTP) and dynamic CTA to evaluate the collateral circulation status of patients with AIS (3840). Therefore, further study will focus on collateral status grading performed on CTA prior to thrombectomy, and thereby a double-check made by DSA, which could have more sense in the development of a reliable score predicting futile recanalization related to the appropriateness and effectiveness of thrombectomy.

In this study, patients in the ANA score 0 group had the lowest rate of futile recanalization (21.05\%), while patients in the ANA groups 2 and 3 had the high risk of futile recanalization (79.03 and $90.91 \%$, respectively). Therefore, according to the ANA score $2-3$ in the present study, these patients may be excluded from the decision-making of thrombectomy treatment although they fulfill the necessary guideline criteria (24). However, in this study, the relatively low AUC, specificity and sensitivity of ANA score were detected, which may be due to the small sample, especially in the division of 140 patients into 4 groups and the further reduced number of patients per group. Secondly, for the thrombectomy performed between 6 and $24 \mathrm{~h}$ from symptom onset in the present study, the different inclusion criteria had been adopted $(17,23,24)$. Furthermore, previous studies reported that female gender and delayed endovascular treatment were also regarded as the independent predictors of unfavorable outcomes in recanalized patients, besides age and NIHSS score $(7,8)$. Thus, further studies with a larger sample size are needed to perform subgroup analysis according to the female gender and procedural time, and to adopt the consistent mismatch criteria between clinical deficit and infarct volume according to the DAWN trial for the late window patients (17), which may contribute to increasing both specificity and sensitivity of ANA score and aid in screening suitable patients for meaningful recanalization much more accurately.

This study was based on actual clinical data. As a retrospective cohort study based on participants from 3 centers, we acknowledged that there were certain limitations. First, the sample size was relatively small. Second, evaluation of the collateral circulation by ASITN/SIR was derived from the invasive DSA examination, which may limit its preoperative application before mechanical thrombectomy. In addition, the relatively low specificity and sensitivity of ANA score, and the different inclusion criteria for the thrombectomy performed beyond $6 \mathrm{~h}$ from symptom onset were shown in this study. At present, there is still a lack of Chinese AIS data on prognosis of thrombectomy in the clinical guidelines, and future researches will be necessary to validate our conclusions by prospective cohort or RCT studies in larger sample of patients.

\section{CONCLUSIONS}

We demonstrate that older age, higher baseline NIHSS score, and lower ASITN/SIR grade are independent risk factors for futile recanalization in AIS patients. The ANA score, which combines patient age, NIHSS score, and ASITN/SIR grade, can effectively predict the risk for futile recanalization. This score may be helpful for selecting potential AIS patients before conducting mechanical thrombectomy. Further studies are necessary to validate the prognostic value of this combined score solely and in combination with other clinical factors for futile recanalization.

\section{DATA AVAILABILITY STATEMENT}

The raw data supporting the conclusions of this article will be made available by the authors, without undue reservation.

\section{ETHICS STATEMENT}

The studies involving human participants were reviewed and approved by Independent Ethics Committee of Shanghai Ninth Hospital, Shanghai Jiao Tong University School of Medicine. Written informed consent for participation was not required for this study in accordance with the national legislation and the institutional requirements.

\section{AUTHOR CONTRIBUTIONS}

JS designed the study and helped revise this manuscript. JL conceived the study and made final approval of this manuscript. $\mathrm{HP}$ and CL analyzed all data and prepared the drafting of this article. LC, YQ, and PH contributed to the acquisition of clinical data. BL and $\mathrm{YZ}$ provided the validation cohort. All authors contributed to the article and approved the submitted version.

\section{FUNDING}

This research was supported by grants from the National Natural Science Foundation of China (81271302 to JL; 82071282 to JS), the research-oriented physicians project II from Shanghai Municipal Education Commission-Gaofeng Clinical Medicine Grant Support (20161422 to JL), the Clinical Research Project from Shanghai Jiao Tong University School of Medicine (DLY201614 to JL), the Biomedicine Key program from Shanghai Municipal Science and Technology Commission (16411953100 to JL), the Rare Disease Registration Platform of Shanghai Ninth People's Hospital, Shanghai Jiao Tong University School of Medicine (JYHJB08 to JS), and the Horizontal Research Project from Shanghai Ninth People's Hospital (JYHX2021001 to JS).

\section{ACKNOWLEDGMENTS}

We acknowledged the specialist editors with suitable professional knowledge who provided professional services for reviewing and correcting the English. 


\section{REFERENCES}

1. Emberson J, Lees KR, Lyden P, Blackwell L, Albers G, Bluhmki E, et al. Effect of treatment delay, age, and stroke severity on the effects of intravenous thrombolysis with alteplase for acute ischaemic stroke: a meta-analysis of individual patient data from randomised trials. Lancet. (2014) 384:192935. doi: 10.1016/S0140-6736(14)60584-5

2. Jauch EC, Saver JL, Adams HPJ, Bruno A, Connors JJB, Demaerschalk $\mathrm{BM}$, et al. Guidelines for the early management of patients with acute ischemic stroke: a guideline for healthcare professionals from the American Heart Association/American Stroke Association. Stroke. (2013) 44:870947. doi: 10.1161/STR.0b013e318284056a

3. Turc G, Bhogal P, Fischer U, Khatri P, Lobotesis K, Mazighi M, et al. European Stroke Organisation (ESO) - European Society for Minimally Invasive Neurological Therapy (ESMINT) guidelines on mechanical thrombectomy in acute ischemic stroke. J Neurointerv Surg. (2019) 11:535-8. doi: 10.1136/neurintsurg-2018-014569

4. Hussein HM, Georgiadis AL, Vazquez G, Miley JT, Memon MZ, Mohammad YM, et al. Occurrence and predictors of futile recanalization following endovascular treatment among patients with acute ischemic stroke: a multicenter study. AJNR Am J Neuroradiol. (2010) 31:454-8. doi: 10.3174/ajnr.A2006

5. Goyal M, Menon BK, van Zwam WH, Dippel DWJ, Mitchell PJ, Demchuk AM, et al. Endovascular thrombectomy after large-vessel ischaemic stroke: a meta-analysis of individual patient data from five randomised trials. Lancet. (2016) 387:1723-31. doi: 10.1016/S0140-6736(16)00163-X

6. Tateishi Y, Wisco D, Aoki J, George P, Katzan I, Toth G, et al. Large deep white matter lesions may predict futile recanalization in endovascular therapy for acute ischemic stroke. Interv Neurol. (2015) 3:48-55. doi: 10.1159/000369835

7. Hussein HM, Saleem MA, Qureshi AI. Rates and predictors of futile recanalization in patients undergoing endovascular treatment in a multicenter clinical trial. Neuroradiology. (2018) 60:557-63. doi: 10.1007/s00234-018-2016-2

8. Hassan AE, Shariff U, Saver JL, Goyal M, Liebeskind D, Jahan R, et al. Impact of procedural time on clinical and angiographic outcomes in patients with acute ischemic stroke receiving endovascular treatment. J Neurointerv Surg. (2019) 11:984-8. doi: 10.1136/neurintsurg-2018-014576

9. van Horn N, Kniep H, Leischner H, McDonough R, Deb-Chatterji M, Broocks G, et al. Predictors of poor clinical outcome despite complete reperfusion in acute ischemic stroke patients. J Neurointerv Surg. (2021) 13:14-8. doi: 10.1136/neurintsurg-2020-015889

10. Pereira D, Fragata I, Amorim J, Reis J. ADC quantification in basilar artery occlusion as an indicator of clinical outcome after endovascular treatment. Neuroradiol J. (2017) 30:586-92. doi: 10.1177/1971400917706197

11. Shi ZS, Liebeskind DS, Xiang B, Ge SG, Feng L, Albers GW, et al. Predictors of functional dependence despite successful revascularization in large-vessel occlusion strokes. Stroke. (2014) 45:1977-84. doi: 10.1161/STROKEAHA.114.005603

12. Xu H, Jia B, Huo X, Mo D, Ma N, Gao F, et al. Predictors of futile recanalization after endovascular treatment in patients with acute ischemic stroke in a multicenter registry study. J Stroke Cerebrovasc Dis. (2020) 29:105067. doi: 10.1016/j.jstrokecerebrovasdis.2020.105067

13. Kawiorski MM, Martínez-Sánchez P, García-Pastor A, Calleja P, Fuentes B, Sanz-Cuesta BE, et al. Alberta stroke program early CT score applied to CT angiography source images is a strong predictor of futile recanalization in acute ischemic stroke. Neuroradiology. (2016) 58:48793. doi: 10.1007/s00234-016-1652-7

14. Gilberti N, Gamba M, Premi E, Costa A, Vergani V, Delrio I, et al. Leukoaraiosis is a predictor of futile recanalization in acute ischemic stroke. $J$ Neurol. (2017) 264:448-52. doi: 10.1007/s00415-016-8366-y

15. Kaginele P, Beer-Furlan A, Joshi KC, Kadam G, Achanaril A, Levy E, et al. Brain atrophy and leukoaraiosis correlate with futile stroke thrombectomy. J Stroke Cerebrovasc Dis. (2021) 30:105871. doi: 10.1016/j.jstrokecerebrovasdis.2021.10 5871

16. Albers GW, Marks MP, Kemp S, Christensen S, Tsai JP, Ortega-Gutierrez S, et al. Thrombectomy for stroke at 6 to 16 hours with selection by perfusion imaging. N Engl J Med. (2018) 378:708-18. doi: 10.1056/NEJMoa1713973
17. Nogueira RG, Jadhav AP, Haussen DC, Bonafe A, Budzik RF, Bhuva P, et al. Thrombectomy 6 to 24 hours after stroke with a mismatch between deficit and infarct. N Engl J Med. (2018) 378:11-21. doi: 10.1056/NEJMoa1706442

18. Son S, Kim YW, Oh MK, Kim SK, Park KJ, Choi NC, et al. Initial factors affecting the clinical outcome after successful recanalization via MR-based mechanical thrombectomy in patients with acute ischemic stroke due to basilar artery occlusion. J Neurointerv Surg. (2016) 8:88993. doi: 10.1136/neurintsurg-2015-011912

19. Yoo AJ, Simonsen CZ, Prabhakaran S, Chaudhry ZA, Issa MA, Fugate JE, et al. Refining angiographic biomarkers of revascularization: improving outcome prediction after intra-arterial therapy. Stroke. (2013) 44:250912. doi: 10.1161/STROKEAHA.113.001990

20. Broderick JP, Adeoye O, Elm J. Evolution of the modified rankin scale and its use in future stroke trials. Stroke. (2017) 48:2007-12. doi: 10.1161/STROKEAHA.117.017866

21. Pexman JH, Barber PA, Hill MD, Sevick RJ, Demchuk AM, Hudon ME, et al. Use of the alberta stroke program early CT score (ASPECTS) for assessing CT scans in patients with acute stroke. AJNR Am J Neuroradiol. (2001) 22:1534-42.

22. Campbell BCV, Mitchell PJ, Kleinig TJ, Dewey HM, Churilov L, Yassi N, et al. Endovascular therapy for ischemic stroke with perfusion-imaging selection. $N$ Engl J Med. (2015) 372:1009-18. doi: 10.1056/NEJMoa1414792

23. Goyal M, Demchuk AM, Menon BK, Eesa M, Rempel JL, Thornton J, et al. Randomized assessment of rapid endovascular treatment of ischemic stroke. N Engl J Med. (2015) 372:1019-30. doi: 10.1056/NEJMoal414905

24. Powers WJ, Rabinstein AA, Ackerson T, Adeoye OM, Bambakidis NC, Becker K, et al. 2018 guidelines for the early management of patients with acute ischemic stroke: a guideline for healthcare professionals from the American Heart Association/American Stroke Association. Stroke. (2018) 49:e46-110. doi: 10.1161/STR.0000000000000158

25. Lee BY, Oh JS, Yoon SM. Long-term prognosis of patients who contraindicated for intravenous thrombolysis in acute ischemic stroke. J Cerebrovasc Endovasc Neurosurg. (2019) 21:77-85. doi: 10.7461/jcen.2019.21.2.77

26. Ghanbari-Homayi S, Dencker A, Fardiazar Z, Jafarabadi MA, MohammadAlizadeh-Charandabi S, Meedya S, et al. Validation of the Iranian version of the childbirth experience questionnaire 2.0. BMC Pregnancy Childbirth. (2019) 19:465. doi: 10.1186/s12884-019-2606-y

27. Jovin TG, Chamorro A, Cobo E, de Miquel MA, Molina CA, Rovira A, et al. Thrombectomy within 8 hours after symptom onset in ischemic stroke. N Engl J Med. (2015) 372:2296-306. doi: 10.1056/NEJMoa1503780

28. Bracard S, Ducrocq X, Mas JL, Soudant M, Oppenheim C, Moulin T, et al. Mechanical thrombectomy after intravenous alteplase versus alteplase alone after stroke (THRACE): a randomised controlled trial. Lancet Neurol. (2016) 15:1138-47. doi: 10.1016/S1474-4422(16)30177-6

29. Dávalos A, Cobo E, Molina CA, Chamorro A, de Miquel MA, Román LS, et al. Safety and efficacy of thrombectomy in acute ischaemic stroke (REVASCAT): 1-year follow-up of a randomised open-label trial. Lancet Neurol. (2017) 16:369-76. doi: 10.1016/S1474-4422(17)30047-9

30. Slivka AP, Notestine MA, Li J, Christoforidis GA. Clinical predictors of cerebrovascular occlusion for patients presenting with acute stroke. J Stroke Cerebrovasc Dis. (2006) 15:303. doi: 10.1016/j.jstrokecerebrovasdis.2005.08.006

31. Zhong W, Geng N, Wang P, Li Z, Cao L. Prevalence, causes and risk factors of hospital readmissions after acute stroke and transient ischemic attack: a systematic review and meta-analysis. Neurol Sci. (2016) 37:1195202. doi: 10.1007/s10072-016-2570-5

32. Smith EE, Kent DM, Bulsara KR, Leung LY, Lichtman JH, Reeves MJ, et al. Accuracy of prediction instruments for diagnosing large vessel occlusion in individuals with suspected stroke: a systematic review for the 2018 guidelines for the early management of patients with acute ischemic stroke. Stroke. (2018) 49:e111-22. doi: 10.1161/STR.0000000000000160

33. Maas MB, Lev MH, Ay H, Singhal AB, Greer DM, Smith WS, et al. Collateral vessels on CT angiography predict outcome in acute ischemic stroke. Stroke. (2009) 40:3001-5. doi: 10.1161/STROKEAHA.109.552513

34. Kidwell CS, Jahan R, Gornbein J, Alger JR, Nenov V, Ajani Z, et al. A trial of imaging selection and endovascular treatment for ischemic stroke. $N$ Engl J Med. (2013) 368:914-23. doi: 10.1056/NEJMoa1212793 
35. Bang OY, Saver JL, Kim SJ, Kim GM, Chung CS, Ovbiagele B, et al. Collateral flow predicts response to endovascular therapy for acute ischemic stroke. Stroke. (2011) 42:693-9. doi: 10.1161/STROKEAHA.110.595256

36. Lau AYL, Wong EHC, Wong A, Mok VCT, Leung TW, Wong KL. Significance of good collateral compensation in symptomatic intracranial atherosclerosis. Cerebrovasc Dis. (2012) 33:517-24. doi: 10.1159/000337332

37. Espinosa de Rueda M, Parrilla G, Manzano-Fernández S, García-Villalba B, Zamarro J, Hernández-Fernández F, et al. Combined multimodal computed tomography score correlates with futile recanalization after thrombectomy in patients with acute stroke. Stroke. (2015) 46:251722. doi: 10.1161/STROKEAHA.114.008598

38. Kim SJ, Noh HJ, Yoon CW, Kim KH, Jeon P, Bang OY, et al. Multiphasic perfusion computed tomography as a predictor of collateral flow in acute ischemic stroke: comparison with digital subtraction angiography. Eur Neurol. (2012) 67:252-5. doi: 10.1159/000334867

39. Jansen IGH, Berkhemer OA, Yoo AJ, Vos JA, Lycklama À, Nijeholt GJ, et al. Comparison of CTA- and DSA-based collateral flow assessment in patients with anterior circulation stroke. AJNR Am J Neuroradiol. (2016) 37:2037-42. doi: 10.3174/ajnr.A4878

40. Di Giuliano F, Picchi E, Sallustio F, Ferrazzoli V, Alemseged F, Greco L, et al. Accuracy of advanced CT imaging in prediction of functional outcome after endovascular treatment in patients with large-vessel occlusion. Neuroradiol J. (2019) 32:62-70. doi: 10.1177/1971400918805710

Conflict of Interest: The authors declare that the research was conducted in the absence of any commercial or financial relationships that could be construed as a potential conflict of interest.

Publisher's Note: All claims expressed in this article are solely those of the authors and do not necessarily represent those of their affiliated organizations, or those of the publisher, the editors and the reviewers. Any product that may be evaluated in this article, or claim that may be made by its manufacturer, is not guaranteed or endorsed by the publisher.

Copyright (c) 2021 Pan, Lin, Chen, Qiao, Huang, Liu, Zhu, Su and Liu. This is an open-access article distributed under the terms of the Creative Commons Attribution License (CC BY). The use, distribution or reproduction in other forums is permitted, provided the original author(s) and the copyright owner(s) are credited and that the original publication in this journal is cited, in accordance with accepted academic practice. No use, distribution or reproduction is permitted which does not comply with these terms. 